\title{
Exclusive Sharing \& Virtualization of the Cellular Network
}

\author{
Timothy K. Forde, Irene Macaluso \& Linda E. Doyle \\ CTVR / The Telecommunications Research Centre, \\ University of Dublin, Trinity College, Ireland. \\ tkforde@gmail.com,macalusi@tcd.ie, ledoyle@tcd.ie
}

\begin{abstract}
The focus of this paper is to progress a discussion about the redefinition of what it means to be a cellular network operator. Alternative modes of ownership may allow for the creation of more flexible cellular networking environments in which competition for packages of resources, i.e. the licenced spectrum, the RAN and core network, ensures that the most efficient architectures win out. This paper introduces the concept of the Cellular Network Cloud and Utility Cellular Networking as a means to explore the possibilities for such revolution in the way in which cellular networks are owned and operated. As a step towards evaluating the technical feasibility of such a paradigm, a flexible auction model that enables us to auction spectrum as a continuous good, as opposed to a collection of discrete items, is presented.
\end{abstract}

\section{INTRODUCTION}

In this paper we are principally concerned with rethinking the concept of resource ownership and resource management in cellular networks. We view cellular networks as entities that can be sliced and diced in new, different and unforeseen ways. The structure of cellular networks, the standards that govern them and the conventional ownership models that are embraced by industry mitigate against innovation and competition in the network's radio access technologies.

As such, the purpose of this paper is to stimulate discussion around what it means to be a cellular network operator. In an environment that is increasingly complex owing to the variety of demands placed on the network infrastructure and abundance of sophisticated techniques that enable networks to be created to address all manner of constraints and objectives, we want to promote the creation of a more flexible network environment in which competition for all resources (spectrum, RAN and core network) ensures that the most efficient techniques are used.

We look to the past to see how and why change has occurred in the ownership and operation of cellular networks to give us insight into how the future cellular network may be owned and operated. If developed and applied to large network infrastructures, dynamic spectrum access techniques offer the opportunity for cellular network entities to exert much more complex control on the network.

The early success of mobile cellular networks was at least based in part on the ability of the various monopolistic network

This material is based upon work supported by Science Foundation Ireland under Grant No. 03/CE3/I405 as part of the CTVR / The Telecommunications Research Centre, University of Dublin, Trinity College, Ireland. operators to exert total control on their resources; the phones available to customers, the RAN which they built out and the PSTN to which, and through which, they connected their customers. But we are now moving from highly integrated architectures to one in which the boundaries between different network entities are now much more blurred and fluid; the nature of user equipment is changing rapidly, the evolution of the radio access technologies is advancing apace and the cellular network value chain is ever changing. To that end, in this paper we look to see if there is an alternative way to own and operate networks such that the resources can be fully exploited by a diverse and competitive group of virtual operators.

The main contribution in this paper includes the exploration of an alternative mode of ownership for future cellular networks. We propose a concept influenced by the Cloud Computing paradigm; the notion of a Cellular Network Cloud that facilitates Utility Cellular Networking is presented. An initial step in the investigation of the technical feasibility of this concept is contributed by way of a new auction model which enables us to auction spectrum as a continuous good, as opposed to a collection of discrete items.

The rest of this paper is laid out as follows. In Section II we will examine the evolution of cellular networks from their inception to date; taking a particular interest in the interplay of technology development and ownership. Section III looks at alternative models of ownership, principally the concept of exclusive sharing. It then goes on to present an overview of Cloud Computing as it relates to our objectives of developing more flexible resource ownership models in the cellular network. In Section IV we develop the concept of exclusive sharing in cellular networks; motivating the extreme virtualization of the infrastructure - the radio networks, the backhaul network and the spectrum. Then, in Section $\mathrm{V}$ we present an example of an auction process that demonstrates increased flexibility over current auction models, a stepping stone towards developing a framework that facilitates the concept of Utility Cellular Networks. Section VI presents a discussion that examines future directions for this work. Section VII concludes this paper. 


\section{InNOVATION, Evolution AND THE MOVEMENT OF OWNERSHIP IN CELLULAR NETWORKS}

If we look to slice, dice and reassemble cellular networks in new and revolutionary ways that take account of DySPAN advances and new ownership structures, we should understand the trajectory of past change in cellular networks. The cellular networks of 2010 differ greatly from the early wireless networks of the 1980s. In that thirty year period change has occurred in the business models, the customer base, the application space and the technologies that drive the networks.

\section{A. Analog, Monopolies}

The first generation of cellular networks can be summarized as follows; fragmented, expensive, revolutionary [1], [2]. There was no global, and little regional, coordination in the rollout of the early cellular networks. Many unique technologies were put in place across the globe. Furthermore, the early cellular networks were designed as extensions of the existing circuit-switched PSTN. They extended the reach of the copper network, enabling wireless voice-based communication by extending the circuit-switched model into the wireless domain.

The concept of the cellular network goes back to 1947 when it was first proposed internally at Bell Labs. The USA, having developed the concept of the cellular phone, gave the world its first successful trials; by 1978 AT\&T had installed 10 basestations in Chicago and by 1981 this test network had reached its 2000 subscriber capacity.

The Advanced Mobile Phone System (AMPS) (at 800MHz) became the de facto standard in the USA as a result of the might of AT\&T/Bell Labs, an organisation with the deep pockets to develop the standard from a conceptual starting point and the business reach across the USA to deploy it widely. In 1979 Nippon Telegraph \& Telephone (NTT) launched its own proprietary services around Tokyo and Osaka.

However, the birth of a true, considered, standard can be traced to 1981, when the 1969-established Nordic Mobile Telephony (NMT), a consortium of the state Postal/Public Telegraph \& Telephone (PTT) organisations of Finland, Denmark, Sweden and Norway, launched NMT-450 (at 450MHz). The NMT-450 system quickly reached capacity and NMT-900 (at $900 \mathrm{MHz}$ ) was launched in 1986 . For whatever reason, France, Germany, the UK and Italy went their own way in developing an analog system choosing to ignore NMT; other, smaller European countries subsequently adopted NMT. In 1986, France rolled out Radiocom 2000 (at 400MHz) ${ }^{1}$. Likewise, Germany, through the state-owned Deutcshe Bundespost and Siemens rolled out C-Netz to $98 \%$ of the West German population in 1986 using the C450 system. This system also incorporated a precursor of the subscriber identity module (SIM) which is a feature of all modern terminals. However, in 1986 this technological feature made the phones

${ }^{1}$ In 1988 France rolled out a NMT-450 service, with slight variations, in parallel with Radiocom 2000. As the system differed from standard NMT-450, no roaming was possible with other NMT-450 systems. more expensive than the comparable NMT handsets, thus less affordable to the masses. As with France's Radiocom, it was not a popular success owing to the expense of accessing the service.

The UK took a different approach to every other European country. Under Margaret Thatcher's leadership the UK was pursuing market-oriented objectives; private enterprise and competition were the order of the decade. In that light, British Telecom (BT) had been spun out from the state-controlled Post Office in 1981 (fully privatised in 1984). BT, as part of a consortium named Cellnet, and a new competitor, Vodafone, were awarded licences to operate cellular services. A joint industry/government group decided on the technology to be adopted by both licencees. While the NMT system was rejected as it could not provide capacity for London, a frequency-adjusted version of US-designed AMPS was adopted and renamed Total Access Communication Systems (TACS). Vodafone were first to provide TACS service in 1984. TACS was also rolled out by Eircell (now Vodafone Ireland), the mobile arm of the Irish state PTT, that year.

Cellular telephony was clearly an innovative technology in the 1980 s, there was nothing like it available to the masses before then.

\section{B. MNOs, Ah Hoc Standards}

The early cellular networks, at least in Europe, were generally built out by the national PTT organisations. In these cases, the network was owned and operated from the RAN through to the PSTN by one monopolistic organisation. Divisions between the cellular network operator (MNO) and the PSTN-operator were non-existent. As such, the network was naturally sold to customers as an extension of the wired telephony service. The monolithic mobile network operator (MNO) prevailed at this point. There was no disruption of the value-chain that already existed for the PSTN.

However, cellular telephony did start to elicit behaviour that would lead to changes in the value chain. In Denmark, an early indicator of the effects of cellular telephony, the detethering of users from their telephone sockets, resulted in a doubling of voice traffic across the Danish telephone system. Technological advances were changing user behaviour.

And, as cellphone users moved, some of them eventually moved out of range of their home networks. The need to allow for roaming was born, but was not anticipated or catered for in the majority of networks. Roaming, to our minds at least, represents the first movement towards virtualization of the cellular network. Even though a customer was in another country, away from its service provider and their billing systems, the service of the home network was replicated for it on the foreign network; calls routed to/from it without changing the telephone number. To the end user of roaming, there was no difference in the service, but possibly a large difference in the price charged for the service. However, roaming was limited to a small subset of operators.

Owing to the tight standardisation of the NMT systems in the Nordic countries roaming was possible. The standard had 
been jointly developed by the 4 states and was implemented by their own PTTs. The fact that NMT was implemented as a standard meant that handsets could be developed for it by any manufacturer privy to the specifications. Consequently, in addition to the domestic Nordic suppliers, phones were also developed in the USA and Japan. This brought the costs of handsets down and created competition in handset formfactor design. Spain, Austria, Belgium, The Netherlands and Luxemburg bought the NMT system for their markets too. The BeNeLux operators created a roaming agreement for their customer base, facilitated by the common NMT infrastructure. The NMT model clearly demonstrated the benefits of standardisation; the economies of scale that can be achieved in manufacturing the infrastructure equipment, the ease of interoperation across networks and the competition that can ensue in the terminal design market.

In the USA, roaming issues stemmed from a different angle. The FCC licenced the early cellular spectrum on the basis of licences which covered Metropolitan Statistical Areas (MSA), i.e. areas which had a significant population to serve. It offered two licences per MSA to stimulate competition; one licence generally went to AT\&T and its post-breakup successors, the other licence went to start-ups. However, the concept of roaming was infrequently provided for. So, even though the USA used the same technology, AMPS, for its network, the licencing process that the FCC engaged in resulted in the assignment of licences to a plethora of companies across the country, creating a geographical patchwork of MNOs. As AMPS was a looser standard, implemented by many different companies across the USA, the configuration of switching equipment to handle roaming customers was complex and avoided. As such, customers were tied to their local networks - limiting the use of the phone.

\section{Digital, Planned Standards}

In the early 1990s, the introduction of digital cellular networks, GSM in Europe and various ones in the USA, marked a step-change for cellular technology and enabled further disruption of the cellular network value chain.

The rush to digital was demanded as a consequence of the failed analog networks of Germany and France. In European terms NMT was clearly a successful transnational system; owing to its widespread adoption and standardized nature it had a comparatively high customer take-up. In 1982, Groupe Special Mondiale (GSM), later Global System for Mobile Communications, was formed by the NMT group and a few other countries that had previously adopted NMT under the auspices of CEPT to explore the feasibility of developing a digital cellular system; one capable of carrying both voice and limited data, i.e. the short message service (SMS). By 1987, GSM has successful prototypes in operation. However, political considerations also played their part in GSM's success. The EU, then the European Economic Community, was ever striving towards an 'ever closer union'. One manifestation of this was the progressive creation of a single market, a market across Europe for the barrier-free movement of goods, services and people. In the 1980s, the actual single market as it exists now did not exist. However, GSM was seen as flag-bearer of this ideal. As such, the European Council (that organ of the EU which represents each member state government) made a recommendation that a common standard be agreed so that unity, through technology, and barrier-free roaming could be demonstrated [3]. And so it was.

In the USA, the demand for digital networks lagged that of Europe; the AMPS system had ably satisfied the market needs. Unlike the situation when AMPS was adopted, the AT\&T behemoth had been dismantled, the cellular network market was already fragmented and so the USA took a free-market approach. In 1992 the FCC decided that both TDMA and CDMA would be eligible candidate technologies for the digital upgrade. TDMA IS-136 (Digital AMPS) was adopted by some carriers, offering a clean upgrade path from the existing AMPS system, protecting the significant investment of incumbents. CDMAOne (IS-95) and GSM were also adopted. So, compared to Europe, the USA had 3 competing technologies. This fragmentation continued to divide the USA and deny the ability to roam.

\section{Breaking the Value Chain: MVNOs}

To understand the nature of the mobile virtual network operator (MVNO) model one has to understand a little about the architecture of the typical cellular network. The cellular network is principally comprised of a radio access network (RAN) and the core network. The RAN consists of the basestations and the terminal devices, i.e. phones. The core network handles billing, switching, roaming, handover, etc. Switching, whether for data or voice, involved the connection of a subscriber's voice call or data session to the PSTN or to the Internet. The MVNO can be defined as a network operator which leases radio access from its hosting MNO. However, the strict definition of, and regulation for, the MVNO differs from country to country [4]. Nonetheless, radio access is needed in order to connect the MVNO's subscribers to the MVNO's switching centre. The MVNO is able to connect its network to the networks of other network operators and to establish interconnection agreements with them.

The key distinction between a MNO and a MVNO is that the MVNO does not own, or control, the spectrum on which it connects to its subscribers.

In Europe at least, MVNOs arose during the lifetime of the GSM networks. MVNOs can come from two starting points; the voluntary and the involuntary. As a limited number of spectrum licences had been awarded in each country, some regulators felt that there was not enough competition, more particularly that there was market control by dominant MNOs. Such regulators intervened to create frameworks which forced MNOs to open their networks to MVNOs; termination charges, wholesale rates were capped by the regulator. On the other hand, voluntary arrangements were also engaged in; according to [5], the following benefits were identified as grounds for a MNO to engage with a MVNO on its network:

- Reach or test new market segments 

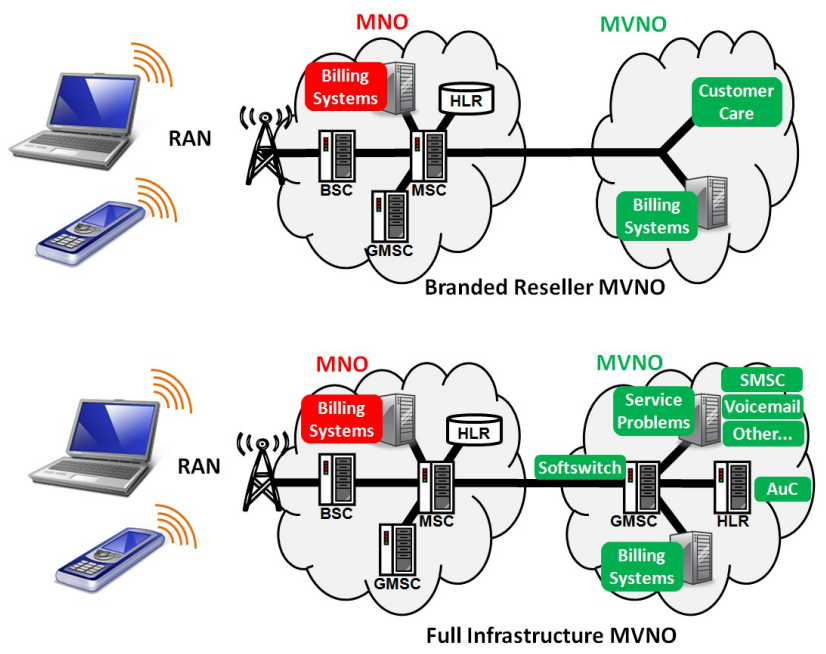

Fig. 1. MNOs and MVNOs: Cellular Network Structure

- Utilize spare network capacity, quickly increase traffic on the network

- Risk sharing by using several service trade marks

- Share network investment costs

- Introduce of new services which supplement existing services

For the MVNO to survive, it has to differentiate itself from the MNO and other MVNOs. The level of differentiation and competition it can engage in depends on how developed it is. MVNOs come in many forms, a few of which are described here, and depicted in Figure 1:

1) Branded resellers. This is the lightest MVNO model, owning very little infrastructure. The reseller just operates a brand, a billing system and the distribution channel. In this model, the MVNO has little bargaining power as the customer. The MNO defines the characteristics of the radio access network and the mobile services that are offered.

2) Full Infrastructure MVNO. In this model the MNO provides the RAN and sometimes part of the core network. The MVNO provides every other element of the cellular network value chain. This model gives the MVNO more power to define its value proposition to the subscriber.

3) Mobile Virtual Network Enabler (MVNE). The MVNE is a middle-man entity that can be positioned between the MNO and the MVNO. Under this model the MNO may just operate the RAN, the MVNE provides all of the intermediate network and business functions, leaving the MVNO to act as purely as the distribution channel. However, by aggregating the demands of several MVNOs, the MVNE can leverage a better deal with the MNO.

So, depending on the level of investment that a MVNO makes, the competition for customers, the control over network resources and the bargaining power moves from one part of the value chain to another as illustrated in Figure 2.

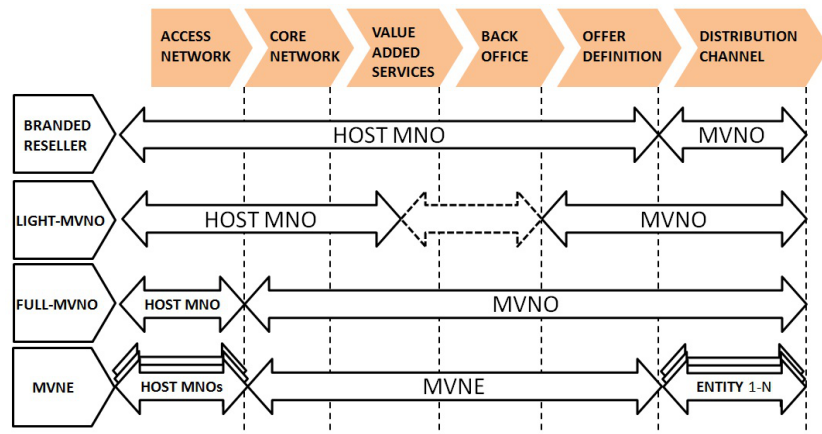

Fig. 2. The cellular network value chain is changing.

As more MVNOs enter the market differentiation on the basis of pricing erodes. However, in the absence of control over the radio access network, service differentiation is difficult as any services offered will be a subset of those possible over the MNO's RAN.

\section{E. IMT-2000: Global Standardization?}

By 1986, before the first digital call had even been made meetings were being convened to plan a third generation of cellular networks. It grew out of the International Telecommunications Union's (ITU) desire to avoid the fragmentation that was obvious in the first and second generations. The aim was to specify a pocket-sized phone that could be used anywhere in the world. Now known as International Mobile Telecommunication (IMT-) 2000, the resulting standard covered 5 different technologies using 3 different radio access techniques; TDMA, FDMA and CDMA. The planning for this standard happened at the same time that the Internet was growing in popularity. Even though data services in the second generation had mixed success (WAP developed as an upgrade to GSM was a failure, i-Mode on Japan's network a success), it was decided to plan for both voice and data services.

IMT-2000 or 3G was initially specified to allow simultaneous voice and data services, with a peak rate of $2 \mathrm{Mbps}$ under restricted conditions. To support this, a new approach to the core network was proposed; separate packet switching and circuit switching components were incorporated. This change in the architecture of the next cellular network reflected the changes occurring in the PSTN which was itself undergoing a significant upgrade as new PSTN network elements shifted to ATM/IP.

IMT-2000 specified a suite of radios that could share the same underlying infrastructure; W-CDMA predominated in Europe/Japan, otherwise known as 3rd Generation Partnership Project's (3GPP) UMTS. CDMA2000 dominated the US and South Korean markets, being an easy upgrade from CDMAOne (IS-95). The standard has evolved over the last decade and as data services have taken off upgrades to the data functionality of the standards have appeared resulting in High Speed Packet Access (HSPA)+ which extends the data capability of WCDMA networks.

$3 \mathrm{G}$ has been a work in-progress, allowing for a smooth 
evolution from the initial technologies which just met the specifications, to the latest upgrade, HSPA+, which boasts a theoretical $56 \mathrm{Mbps}$ in the downlink.

\section{F. Kindles, iPads, Anything USB, M2M}

The significant shift in traffic from voice to data has also unleashed a new wave of terminal devices onto the networks; devices which are driving new MVNO models. Data services, obviously, have a different usage profile to voice services; not being reliant on another human to sustain a communication transaction results in more and different traffic. In the last few years there has been an explosion of new data-only terminals; Apple's iPad, Amazon's Kindle and USB modems that provide data access to laptops, tablets, etc. These are the cutting edge terminal devices of 2010. The Motorola DynaTAC 8000x was the terminal device of 1983.

These devices are driving new relationships between the cellular network operator and the customer. In the case of the Apple iPad, the user requires a data-only service from the network operator. In the case of the Amazon Kindle, Amazon acts as MVNO; when a Kindle is purchased from Amazon it comes with its own contract with the network for which there are no monthly charges. Rather, Amazon pay the wireless fees so that you can buy their books over a cellular network, ostensibly any place, any time - no longer reliant on patchy WiFi coverage. Furthermore, the Amazon MVNO, Whispernet, has roaming agreements in place around the globe. In effect a book retailer has out-sourced the manufacture of a terminal device and provision of cellular network connectivity to shift merchandise from its electronic shelves.

Furthermore, cellular-based machine-to-machine (M2M) communication systems will have their own distinct set of demands depending on the domain in which they are used, i.e. health-monitoring, utility metering etc.

Given these changes in the elements driving demand on the cellular network, and the new kinds of MVNOs that are emerging, it is a very tough task for anyone, or any standards body, to readily predict the future form of the cellular networking industry and the cellular networks it will require.

\section{G. Evolving for the Long Term}

As much as the MVNO models that have been developed to date enable more competition, and more service differentiation in the cellular network, they still stop short of opening the radio access network to competition, innovation and service differentiation. While we look far into the future in terms of the kind of fluid resource ownership that we envisage, the standards that are coming on stream in the near-term provide a basis for the beginning of thought experimentation with new forms or resource ownership and management. The 3GPP UMTS Long Term Evolution(LTE) [6] and its successor LTE Advanced [7] offer a clean slate on which to experiment with new concepts of ownership.
LTE is a supposed greenfield technology, it introduces a new physical layer concept and a new protocol architecture for UMTS which is not compatible with any standard that has gone before it. Its objectives are to achieve high data rates, be low latency and to use packet-optimised radio access technologies. In the core network it embraces the obvious success of IP networking, moving the core network to an allIP solution. More functionality is moved to the basestations, traditionally out of bounds for the MVNO. LTE also requires more spectrum to achieve its target data rates; a $20 \mathrm{MHz}$ channel should theoretically result in $100 \mathrm{Mbps}$ in the downlink.

However, LTE, while fresh and embracing the latest radio techniques and networking technologies only serves to lay the basic building blocks for the proposed future of cellular networking, LTE Advanced - a standard that is currently under development. This standard proposes many technologically exciting and challenging optional upgrade paths from LTE. Among the options that are being explored for LTE Advanced are carrier aggregation, heterogeneous networking (hetnets), single frequency operation, distributed relaying, enhanced MIMO, enhanced scheduling. Each of these techniques represent a major change to the existing form of digital cellular networks. LTE Advanced carrier aggregation will enable the creation of $100 \mathrm{MHz}$ channels by taking component channels from a variety of bands, both contiguous and non-contiguous. This one feature makes LTE Advanced a technology which is fit to exploit a more market-oriented spectrum assignment regime in which many bands of spectrum, as identified by the ITU, are made available. A LTE Advanced network could, theoretically, access spectrum in the $450 \mathrm{MHz}$ band, the 790 $\mathrm{MHz}$ band and the $3.4 \mathrm{GHz}$ band at the same time, for the same cell. The other features, such as hetnets and relays bring network architecture features formerly only associated with experimental networks such as MANETs in to the mix.

To accommodate the anticipated growth in data-focussed cellular networks, the ITU has identified additional spectrum to cope for IMT-Advanced technologies, of which LTE Advanced and IEEE $802.16 \mathrm{~m}$ are two candidates.

\section{Exclusive Sharing - Collaborative CONSUMPTION}

So, the question that arises for us is how we bring competition for control of network resources right into the heart of the cellular network; how can we facilitate the sharing of resources in ways that let different operators control the pieces of the network that they require, given their customers' demands and their abilities to dynamically architect a network.

The concept of sharing expensive resources is not a new one. Outside of the telecommunications domain there have been developments in the exploration of new modes of resource ownership and operation. The exclusive sharing, or collaborative consumption [8], of resources has been in existence for a long time, e.g. car rentals, hotel bedrooms. More recently, the concept of fractional ownership has been used to manage resources such as private aircraft. 
The basic business model of a hotel clearly illustrates the concept of collaborative consumption. This is an industry that invests in expensive real-estate, think Manhattan, builds a durable, luxurious, building containing hundreds of bedrooms and shares those beds among hundreds of thousands of users who otherwise most likely could not afford to either buy a property or take a short-term lease for the time they require to be in Manhattan. While hotels do not market their services as bed-sharing facilities, that is what they are.

The basic premise underlying the exclusive sharing model, whether it is for bed sharing, car rental or aircraft sharing, is that an individual resource consumer cannot afford, or does not wish to invest in, the cost of developing and maintaining a resource for which it will not have a permanent continuous need. The reach of this model has spread in recent times; bike-sharing and car-sharing schemes have cropped up in many cities, private home/bed-sharing industry has grown that uses private homes to provide accommodation for strangers, a modern take on the old-fashioned $\mathrm{B} \& \mathrm{~B}$. In the case of the bike-sharing schemes as operated in Europe ${ }^{2}$, municipal governments have provided advertising space in return for the investment in bike-sharing infrastructure, i.e. bikes, secure bike stands and payment/security mechanisms; city dwellers can then use the bikes for a nominal sum for a limited time. In the case of car-sharing schemes such as Zipcar ${ }^{3}$ and Streetcar ${ }^{4}$ users can access a car by the minute and by the hour, collecting and returning cars on streets convenient to them. The carsharing model is a more fine-grained car (resource) allocation model than the traditional car rental scheme in which rental is by the day and organised from, often inconvenient, car lots. Zimride ${ }^{5}$ is an example of a ride-sharing scheme in which excess capacity, i.e passenger seats in a car going on a specific journey, is made available to other users through the creation of private, social networks that match supply, i.e. free seats, and demand. AirBnB ${ }^{6}$ and Couch Surfing are newer examples of house/bed-sharing schemes. They differ from the hotel model in that people let their private homes, rental properties or a bedroom within their home on a nightly basis to strangers. Nonetheless, the model provides a win for both sides; the host utilizes an unused bed in their home in exchange for cash, and the guest gets a bed at a cheaper rate than at a nearby hotel.

However, there are obvious differences between a hotel and a budget-oriented service such as AirBnB. The AirBnB model uses the furniture that you had, presumably, bought for your own use. The hotel has been designed with repeated use in mind. Unlike a private home, a hotel is built with the intention of sharing rooms repeatedly with strangers. It is built for longevity and it is built to suit the needs of every guest and no particular guest. Hotel rooms are built without personality; they are functional and bland and allow the every guest to feel, somewhat, at home in their own space.

\footnotetext{
${ }^{2}$ http://www.dublinbikes.ie/

${ }^{3}$ http://www.zipcar.com/

${ }^{4}$ http://www.streetcar.co.uk/

${ }^{5} \mathrm{http}: / / \mathrm{www} . z i m r i d e . c o m /$

${ }^{6}$ http://www.airbnb.com/
}

The key point here is that successful collaborative consumption or exclusive sharing schemes are not heavily biased towards any particular user; each user will have different demands so providing the most generic form of the item or service to be shared maximises the likelihood of it being used.

\section{A. Cloud Computing}

Turning to a more technical example of exclusive sharing, Cloud Computing is a concept that addresses many of the ideas we propose for cellular networks. Cloud Computing is about presenting computing as a utility [9], [10]. The advent of Cloud Computing has meant that Internet application developers can bring their ideas to the market without having to make a large financial outlay. The innovative cloud user doesn't have to worry about overprovisioning for something that might fail. Nor to they have to worry about underprovisioning. The US National Institute of Standards and Technology (NIST) defines Cloud Computing as "a model for enabling convenient, on-demand network access to a shared pool of configurable computing resources (e.g., networks, servers, storage, applications, and services) that can be rapidly provisioned and released with minimal management effort or service provider interaction" [11].

Cloud Computing generally refers to the sum of two services; applications that are delivered over the Internet as services and the actual hardware and software in datacentres that provide the services [12]. Three service models have been identified for the cloud [11]; Software as a Service (SaaS), Platform as a Service (PaaS) and Infrastructure as a Service (IaaS), although these categories are seen as somewhat arbitrary, shifting and vague [12]. Each of these three models progressively reveals more of the Cloud's underlying resources to the user. The SaaS model just provides access to applications, and possibly application configuration settings, but does not allow the user access to the configuration of the underlying infrastructure. Whereas the IaaS model concerns the supply of fundamental computing services to the customer such as storage, networking and processing fabrics, allowing the user to install arbitrary software and control the operating systems. The SaaS model compares to the branded reseller MVNO model; the IaaS model has no real equivalent as yet although the MVNE model goes someway to providing core network services in this way to competing MVNOs.

The actual datacentre and the hardware, that is referred to as the Cloud, can be organised in a number of ways depending on the concerns for cost and perceived security issues. Clouds can be Public, Private, Community or Hybrid in form [13]. In the Public Cloud, the service is generally sold on a pay-as-you-go model; utility computing. Resources are dynamically provisioned on a fine-grained, service-dependent basis. Private Clouds refer to data centres internal to large organisations, offering less of the shared capital outlay benefits of the Public Cloud model. The Community Cloud refers to a cloud which serves a number of different organisations; each organisation shares the cost provisioning the cloud but benefits 
from matching. It is harder to reap the full benefits of cloud computing from such a model if the mix of demands on the cloud do not match over time - under and overprovisioning may still occur. The Google U.S. Federal Government Cloud, Apps.gov, is one such example; although its existence may be motivated more by security concerns than cost implications.

Competition between clouds exists, aside from in the form of pricing, in the way in which different utility computing companies differentiate and distinguish themselves based on the levels of abstraction that they present to the programmer, the end-user who creates the service on the cloud, and the way that they manage the resources. Amazon offer two types of services, a high-level web services (AWS ${ }^{7}$ ) platform in the cloud and Amazon EC2 ${ }^{8}$, the Elastic Compute Cloud. AWS provides the typical applications currently found across the Internet; media and entertainment services, search engines, databases and the like. Amazon's AWS is very similar to Google's AppEngine ${ }^{9}$. EC2 offers raw expandable computing power; users pay for CPU units, memory, bandwidth and then execute their own arbitrary applications on the Amazon virtual machine. Microsoft Windows Azure ${ }^{10}$ also offers lower level access to the cloud. Users can write their own applications in Microsoft's .NET, compile them and have them run in a language-independent managed environment.

In [12] it is argued that Cloud Computing was enabled by the construction and operation of extremely largescale, commodity-computer datacentres built at low-cost locations. Such a shared infrastructure led to a decrease, by a factor of at least 5 , in the cost of electricity, network bandwidth, software, hardware, resource management when such large economies of scale were available. When these factors were combined with statistical multiplexing to increase the utilization of the cloud, compared to a private or small community cloud, it resulted in clouds that were able to offer very profitable cloud services. In [12] it is indicated that the following considerations explain the success of Cloud Computing:

1) the illusion of infinite computing resources available on demand.

2) the elimination of an up-front commitment by Cloud users.

3) the ability to pay for use of computing resources on a short-term basis as needed

Cloud Computing succeeds because it creates a submissive ecosystem of datacentres and services onto which innovative developers can imprint their designs, their clever use of the resources. As a model that allows for such innovative use of resources we now turn to see if the Cloud Computing paradigm can be applied, albeit in a modified fashion, in the cellular network world.

\footnotetext{
${ }^{7}$ http://aws.amazon.com/

${ }^{8}$ http://aws.amazon.com/ec2/

${ }^{9} \mathrm{http}: / /$ code.google.com/appengine/

${ }^{10} \mathrm{http}: / /$ www.microsoft.com/windowsazure
}

\section{Extreme Virtualization - The Cellular Network as a Cloud}

As we said at the outset, the aim of this paper is to further discussion around what it means to be a cellular network operator. In an environment that is increasingly complex owing to the variety of demands placed on the network infrastructure and the abundance of sophisticated techniques that enable networks to be created to address all manner of constraints and objectives, we want to promote the creation of a more flexible network environment in which competition for all resources (spectrum, RAN and core network) ensures that the most efficient techniques win out. It may be a utopian vision, but it is one that engages us to rethink the concept of ownership anew.

\section{A. Cellular Network Clouds - Utility Cellular Networking}

In this paper, we want to push virtualization into the most challenging part of the network; into the radio access network, into the control of the spectrum as well as into the core network. We attempt to do this by introducing the concepts of the Cellular Network Cloud and Utility Cellular Networking.

In short, the Utility Cellular Networking proposition is as follows:

1) A Cellular Network Cloud exists: A collection of raw, programmable, versatile equipment is available. This would consist of sites, programmable basestations, microwave links, core network elements, etc.,

2) Each Operating Entity assembles a package of components according to its own (innovative) architectural choices

3) However, unlike the Cloud Computing, the illusion of infinite resources doesn't stand up in the cellular networking domain, necessitating the award of packages of resources (network components) in the Cloud on a competitive pay-as-you-go economic basis.

It was noted in Section III-A that [12] had identified a number of reasons that explain the success of Cloud Computing. We now address these points in light of the limitations of the cellular networking paradigm:

- The illusion of infinite resources. The economies of scale that make Computing Cloud a viable model for Utility Computing are not widely applicable to the cellular networking domain. Coverage in rural areas is still a problem; universal service requirements for geographic or population coverage mandate a minimum coverage which does not leave excess capacity in rural areas. Even though the spread of resources, i.e. basestations, back-haul links, etc., is uneven in geographic terms, the concept can quite readily be applied to dense urban cellular networks where the possibility to create alternative networks is feasible. A simple example is illustrated in the next section. 
- elimination of up-front commitment by Cloud users. The successful examples cited in Section III-A are based on companies that leveraged their existing expertise in largescale data-centre management; these companies were already using their own Private Clouds to run their own enterprises. The services that are offered in the Amazon AWS and Google AppEngine replicate some of the services that they offer under their own branding.

The question as to who would provide the infrastructure for Utility Cellular Networking could be resolved in a number of ways. Existing cellular network infrastructure owners could combine and upgrade their resources to form Community Cellular Network Clouds. Or, alternatively, a public-private partnership model could be engaged whereby government/industry would spur the creation of a truly Public Cellular Network Cloud.

- The ability of pay for resource on a short-term basis. If the concept of Utility Cellular Networking means anything it should mean that the resource user is not constrained to use an arbitrary amount of resources for an arbitrary period of time.

\section{B. Virtualizing the Infrastructure}

Virtualization is the creation of a virtual version of something, rather than the actual thing itself. The distinction between real and virtual in terms of computational entities is not very satisfactory. What is the real difference between a radio implemented in hardware, a so-called real radio, and a radio implemented in software on a general purpose processor, a socalled virtual radio, if they both have the same performance characteristics. But for the purposes of describing the concept of a virtualized cellular network infrastructure let us think of virtual components as being those components that are brought into a useful state by the configuration, reconfiguration, assembly and programming of generic components that would not operate as network components without our intervention. The concept of network infrastructure virtualization goes hand in hand with the design of submissive network components; components which do not have features that bias them towards any particular technology or model of usage, but become valuable network entities when flashed with software or configured in some other way.

In terms of spectrum it means that band plans and prescriptive assignment rules are removed; the spectrum is not biased towards any particular use. A $10 \mathrm{MHz}$ channel of $700 \mathrm{MHz}$ spectrum could just as easily be virtual TV spectrum as it could be virtual WiMAX spectrum. In effect, this is just another way of describing the philosophy of technical- and service-neutral licencing, as espoused by WAPECS [14].

In terms of traditional RAN components such as basestations, it is easily accomplished by deploying completely programmable systems. While the concept of virtualization at the spectrum end of the cellular network may seem challenging, the concept of exclusive sharing at the RAN is not novel. In current 3G (UMTS) systems sharing in the RAN is possible and can occur at different levels; site sharing, i.e. the land used to site basestation equipment (e.g. UMTS NodeB, LTE eNodeB), tower, etc., NodeB sharing and Radio Network Control (RNC) sharing. An advance on this exists in the form of the software-radio basestations which allow multiple standards to be instantiated on them. The flexibility of the software-defined RAN allows for virtualization at this part of the network, such RAN equipment is currently available on the market ${ }^{11}$.

At least two proposals have already been made which approximate to limited instantiations of Cellular Network Clouds. The DIMSUM-net architecture introduces the idea of a Coordinated Access Band (CAB) managed by a spectrum broker into which existing operators could dip if they needed to lease peak-time spectrum in addition to the spectrum they own, or have on a long-term licence [15]. If different uses of the spectrum are allowed for, then statistical multiplexing of access described to such band should enable efficient spatial and temporal use the $\mathrm{CAB}$ spectrum.

In [16], the authors have proposed the concept of radio virtualization, describing a Virtual Radio Network that could be deployed by an infrastructure provider. In this type of virtual radio network, the physical resources are designed and deployed in such a manner that a resource manager can partition and allocate network elements to different virtual network operators.

Aside from these theoretical discussions of radio virtualization, the notion of a quasi-submissive network has already been experimented with; the Rutgers University-based ORBIT [17] network is a laboratory-based wireless network emulator comprising a large two-dimensional grid of 400 802.11 radio nodes which can be dynamically interconnected into specified topologies with reproducible wireless channel models. It also comprises a field trial network, allowing for a mix of configurations involving both $3 \mathrm{G}$ and 802.11 wireless access in a real-world setting.

The concept of virtualization and submissive design extends to every other element of the network; microwave links, fibre links and the switches in the core network. In fact, as both LTE and LTE Advanced move to an All-IP architecture in the core, the transition of these elements of the network to cloudbased core network services would seem to be realisable in the medium-term.

\section{Differentiation}

So, the key feature enabled by this approach is that competing network operator entities can differentiate themselves by offering bespoke solutions to cellular networking demands even though everyone is accessing the same basic resources in the Cellular Network Cloud. Each network entity chooses the package of network components that it wants and configures them in such a way that its desired architecture is realised.

If we take the example of the Cellular Network Cloud depicted in Figure 3, a number of features are evident. There is

\footnotetext{
${ }^{11}$ http://www.vanu.com
} 


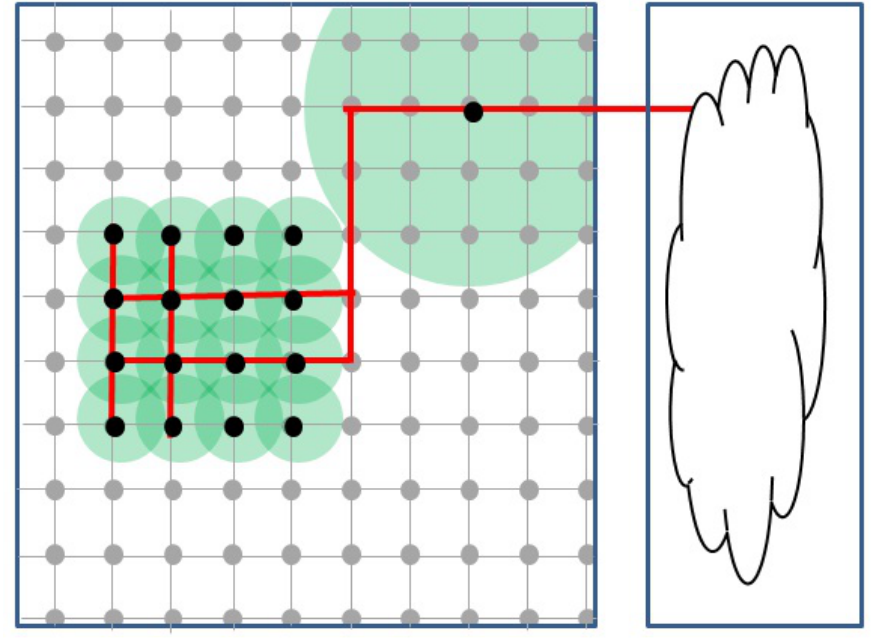

Radio Access Network

Core Network

Fig. 3. Cellular Network Cloud

a dense urban-like concentration of sites, each site equipped with a programmable basestation. There is also a lattice of microwave links, or possibly fibre links, which leads to the core network; these backhaul links are organized in such a way that any combination of them can be chosen to create any path to the core network.

If we assume that the dominant standard is LTE-Advanced, a variety of options are open to each network operator entity in how they architect the network. As noted in Section II-G, LTE-Advanced proposes to allow for heterogeneous networks, distributed relaying, single frequency operation, MIMO operation, etc. The operator's ability to access and schedule resources using the optimum selection of LTE-Advanced would be key. Figure 3 indicates that the operator has chosen to implement a single frequency operation with heterogeneous cell coverage; a collection of small cells for high capacity in one area and a single large cell in another sparsely populated area. Most of the basestations are linked to the core network by means of back-haul connections, but 4 cells are connected by means of relay.

\section{VirTualizing SPECTRUM}

As with each of the other elements that would go into creating a Cellular Network Cloud spectrum is a finite resource, hence the pursuit of DySPAN techniques and policies that allow it to be efficiently used, whether in a licenced or unlicenced context. As such, it is difficult to create the illusion of an infinite supply of licenced spectrum. Rather, it is our view that the economically efficient, least arbitrarily restricted assignment of spectrum approximates that illusion [18]. To that end, in this section we present an auction model that extends the flexibility found in existing auction models, removing yet more restrictions.

Unlike the kind of fine-grained within-assignment virtualization presented in [15], [16], we are interested in virtualizing

the spectrum a step before that, at the point of assigning the spectrum within the market to competing classes of users, technologies and standards. While we view cellular networks as entities that can be sliced and diced in new, different and unforeseen ways, the model we present here would also allow for the assignment of spectrum to operators of a DIMSUMnet-like system [15] or a virtual radio network [16] who themselves sub-virtualize the spectrum to provide different types of cloud services.

It has been shown that the more expressive a market is, the more Pareto efficient it is [19]. An expressive market is one that allows buyers and sellers to ask, in so far as it can be accommodated in the market mechanism, for whatever they want, specifying constraints and conditions that suit their requirements.

In terms of spectrum markets, combinatorial auctions enable buyers to stipulate that they require certain packages of spectrum, e.g. an FDD operator requires both uplink and downlink spectrum, not just one link. But such freedom is in practice curtailed; regulators and market incumbents are slow to embrace expressive, flexible auctions. Two kinds of inflexibility can be identified in the approaches to spectrum auctions that have been implemented or proposed so far. In all cases, spectrum auctions are based on the discretization of the spectrum. The spectrum is divided in blocks of fixed size and bidders ask for specific blocks, if the Simultaneous Multiple Round Ascending (SMRA) auction format is adopted, or for an amount of blocks in the case of a combinatorial clock auction (CCA).

We believe that the flexibility provided by dynamic spectrum access techniques, cognitive radios, software radios and technologies beyond LTE-Advanced will enable us to auction the spectrum as a continuous good, as opposed to a collection of discrete items. Fixing a priori the size of the base unit of spectrum is equivalent to restricting the bundles on which bids can be submitted. This may limit the possible market outcomes and, consequently, it may result in economic inefficiencies.

The second kind of inflexibility is related to the band plan assumed for the auction process. Usually the auctioneer decides in advance a fixed allocation of the spectrum between different technologies, in particular between paired and unpaired spectrum. The original Ofcom proposal for the $2.6 \mathrm{GHz}$ spectrum award [21] partially addressed this limitation. However, this approach, as well as the Dutch $2.6 \mathrm{GHz}$ auction which is based on it [22], does not allow for asymmetric requests of paired spectrum; a change necessary to facilitate cellular networks such as LTE-Advanced.

Our model overcomes these limitations. Bidders can ask for amounts of spectrum, as opposed to a number of fixedsize spectrum blocks. Moreover, both flexible band plans and asymmetric requests of paired spectrum are supported. A further improvement of the proposed model is the auction of spectrum in different bands, locations and time periods simultaneously, as illustrated in figure 4.

It should be noted that enabling freer expression for the buyer, which may be seen as simplifying the bid process, 


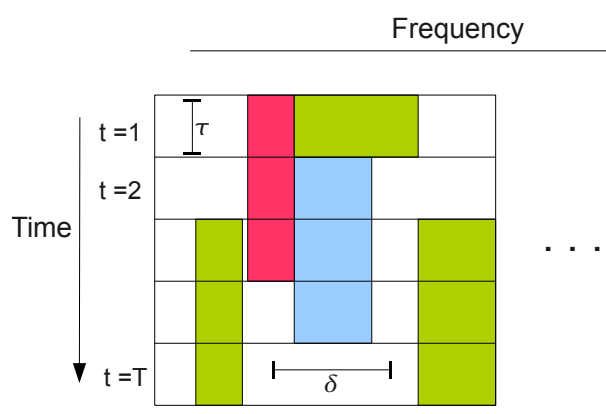

$B_{1}$

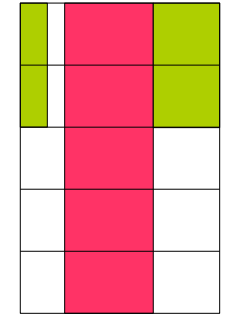

$\mathrm{B}_{\mathrm{m}}$
Fig. 4. Different colors represent different bids. Bidders can ask for paired and/or unpaired spectrum in one or more band/location $B_{j}$.

can increase the complexity of the auction process itself, specifically when it comes to the final stages which address the winner determination problem (WDP).

Analogously to what was proposed in [21] the spectrum auction consists of two stages: the auction and the assignment stage. During the auction stage, the CCA format is adopted. Each band/location/period category has its own "clock price". At each round, bidders ask for a combination of paired and/or unpaired spectrum in one or more category. The clock price of each band/location/period is incremented if there is an excess of demand. The auction stage ends with the determination of the winning bids. In the assignment stage, the actual allocation of frequencies for each band/location/period is determined. Once the winning bids are determined, many possible allocations are possible. To limit the interference between adjacent systems, the assignments will be contiguous. For the same reason, paired and unpaired spectrum channels will be grouped as illustrated in figure 5. Unpaired spectrum is assigned in the centre of the band so as to ensure that duplex spacing between paired channels is adequate. Moreover the assignments in the uplink and downlink spectrum follow the same order.

\section{A. Model description}

The auctioneer has a set of bands in different locations, $\left\{B_{1}, B_{2}, \ldots, B_{m}\right\}$, to sell. A band $B_{j}$ is auctioned for a limited period of time $\tau$, and the auction covers $T$ periods. A bidder can ask for a channel in the band $B_{j}$ for $t^{\prime} \leq T$ periods. The periods requested by the bidder do not need to be contiguous (see figure 4). In the following, we will denote by $B_{j t}$ the band $B_{j}$ in period $t$, where $t \in\{1,2, \ldots, T\}$. A package bid is a combination of channels in different locations, bands and time periods, and the corresponding price, $p_{i}$, the buyer is willing to pay for it. The winner determination problem computes which bids are to be accepted in order to maximize the auctioneer revenue under the constraint that the allocated channels do not exceed the available spectrum in each band/location/period.

The model for the winner determination problem has to take into account not only the availability of spectrum, but also the requirements connected to the allocation of paired and unpaired spectrum in a certain band. In particular, a constraint on the duplex spacing for paired spectrum is required to avoid harmful self-interference.

Let us denote by $C_{i t}=\left\{c_{i 1 t}, c_{i 2 t}, \ldots, c_{i m t}\right\}$ the set of channels requested in the $\mathrm{i}$-th bid, where $c_{i j t}$ is the amount of spectrum in the $\mathrm{j}$-th band/location and at period $t$. No limitations are imposed on the bid packaging, and a bidder can ask for both paired and unpaired spectrum in each band. The combinatorial clock auction we designed would not require the bidder to distinguish paired and unpaired spectrum, as the excess is computed for each band as a whole. However, to guarantee that the duplex spacing constraint is satisfied, $c_{i j t}$ has to be specified in terms of the amount of unpaired $\left(c_{i j t}^{u n}\right)$, uplink $\left(c_{i j t}^{u l}\right)$ and downlink spectrum $\left(c_{i j t}^{d l}\right)$ that is requested for each band/location/period.

The WDP can be formulated as follows:

$$
\begin{aligned}
& \max \sum_{i=1}^{n} p_{i} x_{i} \\
& \text { s.t. } \\
& \sum_{i=1}^{n} c_{i j t} x_{i} \leq B_{j}, \quad \forall j \in\{1,2, \ldots, m\}, \forall t \in\{1,2, \ldots, T\} \\
& \sum_{i=1}^{n} c_{i j t}^{u l} x_{i}+\sum_{i=1}^{n} c_{i j t}^{u n} x_{i} \geq \delta_{j}, \\
& \qquad \forall j \in\{1,2, \ldots, m\}, \forall t \in\{1,2, \ldots, T\}
\end{aligned}
$$

$x_{i} \in\{0,1\}$.

The first constraint in (1) guarantees that the allocated spectrum does not exceed the available spectrum for each band/location/period. To simplify the notation, we will omit in the following the subscripts $j$ and $t$. If the assignment stage is performed as explained in section $\mathrm{V}$ and if we assume that $c_{i}^{d l} \geq c_{i}^{u l}, \forall i \in\{1, \ldots, n\}$, the second constraint in (1) ensures a minimum distance $\delta$ between paired spectrum channels. For paired spectrum channels $i$, the distance between uplink and downlink band is:

$d_{i}=d_{i}\left(c_{i}^{u l}, c_{i}^{d l}\right)=c_{i-1}^{d l}+c_{i-2}^{d l}+\ldots+c_{1}^{d l}+U n+c_{k}^{u l}+\ldots+c_{i}^{u l}$

where $U n$ is the amount of allocated unpaired spectrum and $k$ is the number of allocated paired channels. As $c_{i-1}^{u l} \leq c_{i-1}^{d l}$, $d_{i-1} \leq d_{i} \forall i \in\{1, \ldots, n\}$. Therefore, the minimum distance between paired channels is $d_{1}=U n+c_{k}^{u l}+\ldots+c_{1}^{u l}$, i.e. the right hand-side of the second constraint in 1 . As $d_{1}$ is the total amount of allocated unpaired and uplink spectrum, this constraint is independent on the frequency assignment order of both paired and unpaired spectrum, as long as the unpaired spectrum is allocated in the centre of the band. In other words, the winning determination problem (1) can guarantee a minimum duplex spacing without dealing explicitly with the assignment of frequencies. Thisis of the utmost importance as it allows the auction of generic spectrum channels, instead of forcing the bidders to ask for specific frequencies.

It is well known that problem (1) is NP-complete. Although the WDP is formulated as a branch-on-bids search problem, 


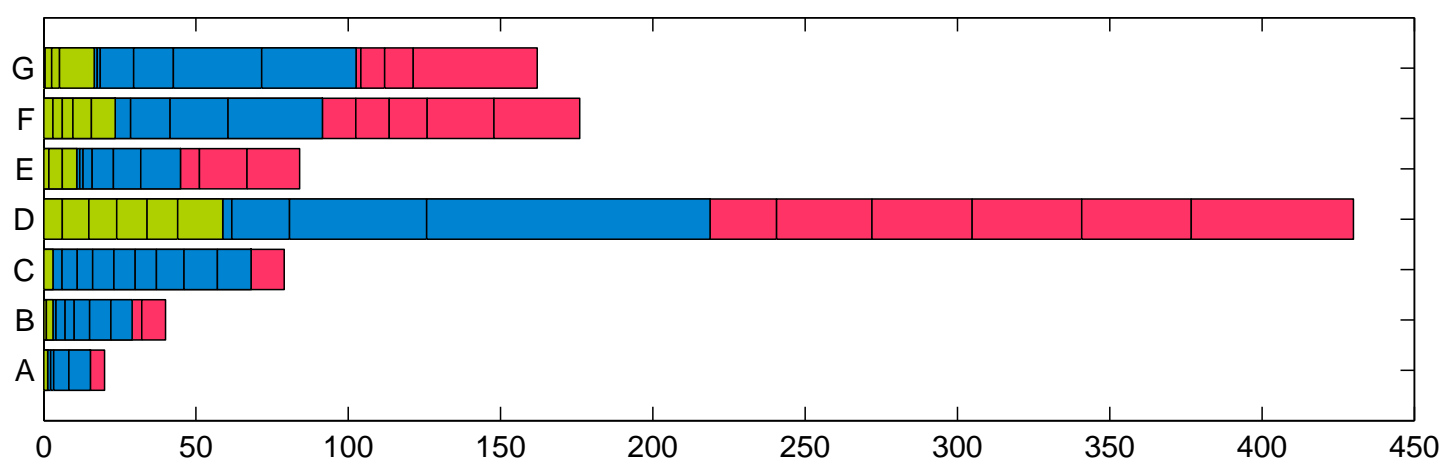

Fig. 6. Illustration of a final assignment across 7 bands; uplinks (green), corresponding downlinks (red) and unpaired spectrum (blue).

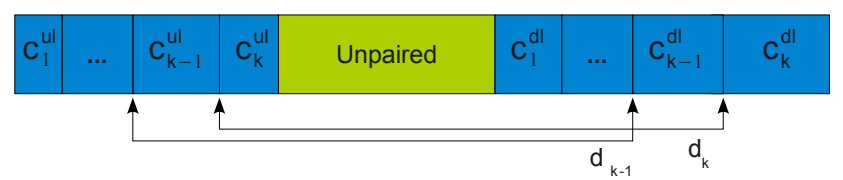

Fig. 5. Unpaired spectrum is assigned in the centre of the band so as to ensure that duplex spacing between coupled paired channels is adequate.

TABLE I

\begin{tabular}{|c|c|}
\hline & ITU Band \\
\hline A & $450-470 \mathrm{MHz}$ \\
\hline B & $790-862 \mathrm{MHz}$ \\
\hline C & $880-960 \mathrm{MHz}$ \\
\hline D & $1710-2170 \mathrm{MHz}$ \\
\hline E & $2300-2400 \mathrm{MHz}$ \\
\hline F & $2500-2690 \mathrm{MHz}$ \\
\hline G & $3400-3600 \mathrm{MHz}$ \\
\hline
\end{tabular}

the specialized search formulation proposed in [20], which is exponential with respect to the number of items but polynomial in the number of bids, cannot be used as in this case the good to be sold is not a collection of discrete items. Some instances of (1) may require too long to get the optimal solution. However, if an anytime search algorithm is adopted, a feasible solution can be obtained by terminating the algorithm after a certain amount of time.

We investigated the potential feasibility of the proposed approach by modeling the spectrum auction for proposed LTEAdvanced networks. We chose this particular scenario because of the high spectrum flexibility supported by LTE-Advanced systems. In particular, the possibility of inter-band carrier aggregation is one of the key features of LTE-Advanced. Moreover, the radios will also be able to operate on both paired and unpaired spectrum, and with variable bandwidths. Table I shows the bands destined to IMT-Advanced technologies.

In the simulation, we considered 5 different locations corresponding to geographical areas where different demands of spectrum are likely to be present. The simulated auction covers a period of 5 years, each divided in 2 semesters (i.e. $T=10$ ). We ran 50 random instances of (1) for $n \in\{50,100,150,200\}$ bids using a state of the art mixed integer program solver, namely CPLEX. For each instance a bid is generated so as to have a minimum and a maximum amount of total spectrum requested along the bands specified in table I. The minimum and the maximum amount of total spectrum requested in a bid depends on the location. For example, in the case of a metropolitan area, the maximum amount of spectrum that can be requested is $140 \mathrm{MHz}$. Although LTE-advanced radio's limit for spectrum aggregation is $100 \mathrm{MHz}$, it is likely that a network operator would require a larger total bandwidth to guarantee the $1 \mathrm{Gbit} / \mathrm{s}$ peak data rate in a densely populated area. To illustrate the assignments that resulted in these auctions, figure 6 depicts a single WDP result. The assignment of paired and unpaired spectrum can clearly be seen in the 7 IMT-Advanced bands; green and red indicate the uplink and downlink, respectively, of the paired spectrum, blue indicates unpaired spectrum.

\section{Discussion \& FUtURE WORK}

The paper has taken us from the monopolistic and highly integrated analog networks of the early 1980s through to the concept of a Cellular Network Cloud. However, we have only begun to scratch the surface regarding the policy and technical issues that need to be addressed to realise a network approximating the Utility Cellular Networking concept. In the next few subsections, we describe the next pieces of work to be addressed in future.

\section{A. Cellular Network Design for Sharing}

As we discussed in Section III, resources that are intended to be used for sharing should be designed in such a way they are not biased for or against any particular use, in so far as that is practical. The concept of submissive component design will be investigated; specifically the limits to which one can take generic design of network components without compromising the efficacy or efficiency of the component. 


\section{B. Assigning Spectrum \& Infrastructure}

It is intended to develop a specialized search algorithm to solve problem (1) more efficiently. We also intend to explore the trade-off between the eventual inefficiencies caused by an early termination of the optimization and the degrees of freedom provided by this model. Further to this, we intend to look at the joint allocation of spectrum and network resources, i.e. base stations, backhaul links, by developing appropriate network and auction models.

\section{Virtualisation \& Standards}

Innovation and standardisation are not good bed-fellows. Standards are borne out of consensus, manipulation by industry and caution. Any wireless networking standard documentation stands as a testament to that statement. Innovations, where they slip by committees and approval ballots, appear as optional extras that may be implemented, but rarely are. In an environment that supports innovation on the part of individual network entities, the role of standardisation will change. LTE Advanced appears to be a standard that will unleash technologies and architectures that may take divergent evolutionary tracks. Issues of backwards compatibility and system interoperability should be investigated in the context of highly virtualized network infrastructure.

\section{CONCLUSION}

In this paper we were concerned with radically rethinking the concept of resource ownership and resource management in cellular networks. As such, one of the purposes of this paper was to stimulate discussion around what it will mean to be a cellular network operator in a future dominated by rich and complex standards such as LTE Advanced and its successors. The paper focussed on an alternative system of ownership for cellular networks, namely one based on the concept of exclusive sharing of common, programmable resources. Taking our cues from the paradigm of Cloud Computing, the concepts of Cellular Network Clouds and Utility Cellular Networking were introduced. A novel spectrum auction model was presented which addressed, in part, some of the technical feasibility issues associated with realising this new ownership model.

\section{REFERENCES}

[1] J. Agar, "Constant Touch - A Global History of The Mobile Phone,"Icon Books, Thriplow, UK, 2005.

[2] K. Tanaka, "The Mobile Communications Environment in the European Union: Systems, Regulations, and Consequences in the Past, Present, and Future", 2001 IEEE Conference on the History of Telecommunications, Rutgers University, USA, 2001.

[3] Communication from the Commission on the Coordinated Introduction of the Pan-European Digital Cellular Mobile Communications System (Report on the Implementation of Council Recommendation 87/371/EEC and Council Directive 87/372/EEC). COM (90) 565 final, 23 November 1990.

[4] Byung Woon Kima \& Seong Ho Seol, "Economic analysis of the introduction of the MVNO system and its major implications for optimal policy decisions in Korea", Telecommunications Policy, Volume 31, Issue 5, June 2007, pp: 290-304.

[5] "MVNO Pricing Structures in Finland", Finnish Ministry of Transport and Communications, Helsinki, 2005.
[6] 3rd Generation Partnership Project (3GPP), Technical Standard 25.913; Requirements for E-UTRA and E-UTRAN (Release 7).

[7] 3rd Generation Partnership Project (3GPP), Technical Specification Group Radio Access Network; Feasibility study for further advancements for E-UTRA (LTE-Advanced), Release 9; 3GPP TR $36.912 \mathrm{~V}$ 9.1.0, December 2009.

[8] R. Botsman, R. Rogers, "What's Mine Is Yours: The Rise of Collaborative Consumption”, HarperBusiness, September 14, 2010, ISBN-13: 978-0061963544.

[9] R. Mikkilineni, V. Sarathy, "Cloud Computing and the Lessons from the Past", Proceedings of the 2009 18th IEEE International Workshops on Enabling Technologies: Infrastructures for Collaborative Enterprises, WETICE '09, 2009, pp. 57-62.

[10] M.A. Vouk, "Cloud computing Issues, research and implementations," Information Technology Interfaces, 2008. ITI 2008. 30th International Conference on , vol., no., pp.31-40, 23-26 June 2008 doi: 10.1109/ITI.2008.4588381

[11] P. Mell, T. Grance, "The NIST Definition of Cloud Computing", Version 15, National Institute of Standards and Technology, Information Technology Laboratory, USA, October, 2009.

[12] M. Armbrust, A. Fox, R. Griffith, A.D. Joseph, R. Katz, A. Konwinski, G. Lee, D. Patterson, A. Rabkin, I. Stoica, and M. Zaharia."Above the Clouds: A Berkeley View of Cloud Computing", Technical Report EECS-2009-28, EECS Department, University of California, Berkeley.

[13] R. L. Grossman, "The Case for Cloud Computing," IT Professional, vol. 11, no. 2, pp. 23-27, Mar./Apr. 2009, doi:10.1109/MITP.2009.40

[14] Radio Spectrum Policy Group, OPINION ON Wireless Access Policy for Electronic Communications Services (WAPECS) (A more flexible spectrum management approach) FINAL, November 23, 2005.

[15] Buddhikot, M.M.; Kolodzy, P.; Miller, S.; Ryan, K.; Evans, J.; , "DIMSUMnet: new directions in wireless networking using coordinated dynamic spectrum," World of Wireless Mobile and Multimedia Networks, pp. 78- 85, 13-16 June 2005.

[16] J. Sachs and S. Baucke, "Virtual radio: a framework for configurable radio networks", in Proceedings of the 4th Annual International Conference on Wireless Internet (WICON '08), ICST, Brussels, Belgium, 2008.

[17] D. Raychaudhuri, I. Seskar, M. Ott, S. Ganu, K. Ramachandran, H. Kremo, R. Siracusa, H. Liu, and M. Singh, Overview of the ORBIT radio grid testbed for evaluation of next-generation wireless network protocols, IEEE Wireless Communications and Networking Conference, March 2005.

[18] L. Doyle and T. Forde, "Towards a Fluid Spectrum Market for Exclusive Usage Rights", IEEE International Symposium on New Frontiers in Dynamic Spectrum Access Networks (DySPAN'07), Dublin, Ireland, 17 - 20 April, 2007.

[19] T. Sandholm, "Expressive Commerce and Its Application to Sourcing: How We Conducted \$25 Billion of Generalized Combinatorial Auctions," Artificial Intelligence Magazine, vol. 28, no.3, 2007, AAAI Press.

[20] T. Sandholm and S. Suri, "Cloud computing Issues, research and implementations," Artificial Intelligence, vol. 145, no.1-2, pp.33-58, Elsevier.

[21] Ofcom, "Award of available spectrum:2500-2690 MHz, 2010-2025 MHz," 2008, UK.

[22] DotEcon and Marsden, R. and Sexton, E. and Siong, A., "Fixed or flexible? A survey of $2.6 \mathrm{GHz}$ spectrum awards", 2010, UK. 